\title{
Molecularly engineered switchable photo-responsive membrane in gas separation for environmental protection
}

\author{
Aishah Rosli ${ }^{\dagger}$, Siew Chun Low ${ }^{\dagger}$ \\ Biomass School of Chemical Engineering Campus, Universiti Sains Malaysia, 14300 Nibong Tebal S.P.S. Penang, Malaysia
}

\begin{abstract}
In recent years, stimuli-responsive materials have garnered interest due to their ability to change properties when exposed to external stimuli, making it useful for various applications including gas separation. Light is a very attractive trigger for responsive materials due to its speedy and non-invasive nature as well as the potential to reduce energy costs significantly. Even though light is deemed as an appealing stimulus for the development of stimuli-responsive materials, this avenue has yet to be extensively researched, as evidenced by the fewer works done on the photo-responsive membranes. Of these, there are even less research done on photo-responsive materials for the purpose of gas separation, thus, we have collected the examples that answer both these criteria in this review. This review covers the utilisation of photo-responsive materials specifically for gas separation purposes. Photo-chromic units, their integration into gas separation systems, mechanism and research that have been done on the topic so far are discussed.
\end{abstract}

Keywords: Gas separation, Membrane technology, Photo-responsive, Stimuli-responsive

\section{Introduction}

Even though various conventional processes have been utilised for the purpose of gas separation [1-4], membrane processes have emerged and remained as an attractive alternative [5-11]. In fact, membranes have managed to make its way into the industry in larger-scale applications [12-16], proving its capability to match the performances of the conventional processes while overcoming their myriad disadvantages. For example, the efforts from Du Pont Co. [12] and Ube Industries [17] justify the potential of membrane in gas separation. This sparks the interest to further improve the membrane processes to yield higher productivity by introducing reversibly switch functional materials in the membrane for a more convenient and energy-saving process [18-21]. The aim is to go beyond the host-guest interaction related stimuli, whereby researchers are intent to expand the concept of the responsive framework to the structural transformation controlled by the external stimuli [22, 23]. Indeed, it opens possibilities to implant a unique functionality in a membrane.

Recent research is geared towards exploiting stimuli-responsive materials whose interfacial properties and subsequent separation

This is an Open Access article distributed under the terms of the Creative Commons Attribution Non-Commercial License (http://creativecommons.org/licenses/by-nc/3.0/) which permits unrestricted non-commercial use, distribution, and reproduction in any medium, provided the original work is properly cited.

Copyright (C) 2020 Korean Society of Environmental Engineers performances can be controlled by external stimuli such as the changes of temperature, light, $\mathrm{pH}$, solution ionic strength, chemical cues or electric and magnetic fields [18, 24-26]. By integrating the stimuli-responsive functional groups in the pores or on the surface of a membrane, the resulting stimuli-responsive membrane can take advantage of the interplay among the membrane pores. Thus, it results in changes of the structural conformation of a membrane or the reactivity of the stimuli-responsive groups to control the mass-transfer and separation processes of a membrane [27]. A trait of a stimuli-responsive membrane that is very appealing is the high rate and selectivity with which the reversible changes occur [15, 28]. For example, Bassanetti and co-workers [29] has successfully produced a flexible crystalline prototypal structure for reversible $\mathrm{CO}_{2}, \mathrm{CH}_{4}$ and Xe uptake. In the study, the expandable porous adamantoid framework manifested its response to selected gasses to promote reversible conformational changes, including enlargement of the channel cross-section, which can thus be considered for eco-friendly gas capture and separation.

A unique thermo-responsive gating adsorption behaviour that depends on the thermal motion of the extra-framework counterions of metal-organic framework (MOF) was presented by Gao and co-workers [30] to observe selectivity uptake of $\mathrm{CO}_{2}$ over $\mathrm{N}_{2}, \mathrm{H}_{2}$,

Received March 5, 2019 Accepted June 10, 2019

${ }^{\dagger}$ Corresponding author

Email: aishahrosli@usm.my (Rosli), chsclow@usm.my (Low)

Tel: +604-599-6412 Fax: +604-599-6908

ORCID: 0000-0002-1827-5665 (Low), 0000-0002-7448-5675 (Rosli) 
and Ar. The $\mathrm{NO}_{3}{ }^{-}$ions in thermos-responsive $\mathrm{MOF}$, $\left[\mathrm{Cu}_{2}(\mathrm{BTR})_{2}\right] \cdot 2 \mathrm{NO}_{3} \cdot \mathrm{x} \mathrm{G}(1)$, located around the adsorption pores exhibited intense motion of the nitrate from closed pore to open pore structure in response to the thermal stimulus. Subsequently, this regulated structural transition from closed to open pore state allowed the passage of specific gas penetrant. At $195 \mathrm{~K}$, results showed no obvious sorption of $\mathrm{CO}_{2}$, implying the closed pore state of MOF. In contrast, an abrupt increase in $\mathrm{CO}_{2}$ uptake was observed when temperature was approaching $231 \mathrm{~K}$, referring to the "opened pores" structure which was dynamically accessible to $\mathrm{CO}_{2}$ molecules. Without causing deformation of the nested structure, authors have demonstrated a reversible gating adsorption by thermally triggering the displacement of the $\mathrm{NO}_{3}{ }^{-}$around the MOF pores, thus enabling a strategic separation and encapsulation of $\mathrm{CO}_{2}$.

However, designing a functional stimulus-responsive material still poses a challenge as they require complicated coordination between the responsive material and the stimulus to get an output that can be measured [31-33]. The preservation of responsiveness function after incorporation on surfaces, at interfaces or in the material itself is key [34]. Thus, having in-depth knowledge of the interacting mechanisms between the stimulus and the corresponding responsive materials can be useful in outlining the inherent physicochemical properties of the responsive materials. This widens the opportunity to further manipulate the performance of the material to explore the many possibilities for designing smart responsive systems. However, as the subsequent sections of this review will reveal, works on photo-responsive systems for gas separation are limited. Other than the lack of understanding the molecular-level mechanism of photo-responsivity that causes the changes in photo-chromic units upon exposure to light, researchers have encountered obstacles such as the degradation of the photo-chromic unit [21] and reduction in the system's performance due to the incorporation of photo-chromic unit [35].

In this review, the synthesis and utilisation of stimuli-responsive materials that undergo physicochemical changes when exposed to external stimuli for gas separation are highlighted. Several reviews have already broached the topic of stimuli-responsive materials in membrane system, however, those mainly focused on liquid-phase separation. Very few have focused exclusively on stimuli-responsive materials for the purpose of gas separation, especially for light- or photo-responsive ones. From this review, some of the limitations in photo-responsive systems for gas separation are presented and addressed, such as the possible mechanisms behind photo-reaction, the less common photo-chromic units as well as the common ones used for gas separation and incorporation of these photo-chromic units into a system that is suited for gas separation. A better in-depth look for a more specific light- and photo-responsive-based membrane system can be obtained in this work, which could possibly spark innovation for further improvement in smart membrane for gas separation processes.

\section{Stimuli-Responsive Materials in Gas Separation}

In past decades, significant progress has been reported in the funda- mental understanding of organic molecules which are capable of reversibly transforming into two or more stable states in response to external signals (temperature, $\mathrm{pH}$, light, etc.) [36]. This opens the possibility of designing sophisticated responsive membrane tools by modifying the chemical composition, design and morphology of a membrane, basing the response mechanism on disparities in surface energy, polymer entropy and segmental interactions [15]. For instance, surface energy is the driving force of the surface responsive reorientation as systems fundamentally minimise the interfacial energy between the surface of the responsive material and its immediate environment. Numerous researches were reported by utilising MOF [24, 37] and membranes [38, 39] for the purpose of gas separation, focusing on the interfacial energy between the responsive material and its immediate environment to control the pore's opening of the separation system to the specific size of gas molecule. Since many gas mixtures to be separated are similar in size, common sieving mechanisms cannot efficiently cater to the specific size of gases. This is where stimuli-responsive materials, with the ability to fine-tune their properties with external stimulus, come into the picture.

In example, Ma and co-workers [40, 41] reported a mesh-adjustable molecular sieve (MAMS) based on a MOF, whose mesh can be adjusted between 2.9 and $5.0 \AA$, which covers the size range of almost all common gas separations. Size adjustment is based on the degree of dehydration of MAMS at various temperatures, which can show temperature-triggered capture and release of specific gas penetrant in a narrow temperature range through designed stimuli-responsive gating effects. To make a MAMS, Ma and co-workers used an amphiphilic ligand 5-tert-butyl-1,3-benzenedicarboxylate (BBDC), [40]) and 4'-tert-butyl-biphenyl-3,5-dicarboxylate (BBPDC, has one more phenyl ring than BBDC), [41] that consists of hydrophobic and hydrophilic ends. The hydrophilic end of the ligand will bind with the metal clusters, where the structure will propagate into a 2D layer. Two layers of ligands will sandwich a metal-cluster layer, thus giving rise to a trilayer packed through van der Waals interaction.

In the study [40], MAMS exhibited highly selective uptake of $\mathrm{H}_{2}$ over $\mathrm{CO}, \mathrm{N}_{2}$, or $\mathrm{O}_{2}$. The molecular sieving effect most likely comes from the BBDC gates. At $77 \mathrm{~K}$, MAMS gate opening was around 3.0 to $3.4 \AA$, with all hydrophilic channels and hydrophobic chambers connected, giving rise to a $3 \mathrm{D}$ gas container with space continuity. Due to the larger kinetic diameters of 3.46, 3.64, and $3.76 \AA$ for $\mathrm{O}_{2}, \mathrm{~N}_{2}$, and CO, respectively, MAMS only allows $\mathrm{H}_{2}$ to enter the hydrophobic chambers with one-dimensional gated channels, which accounts for the high $\mathrm{H}_{2}$ uptake using MAMS at $77 \mathrm{~K}$. When the temperature was raised to $87 \mathrm{~K}$, MAMS can take up a significant amount of $\mathrm{O}_{2}$. In fact, if temperatures can be tuned continuously and precisely, gas separations such as those of $\mathrm{N}_{2} / \mathrm{O}_{2}$, which is difficult to achieve because the size difference of these two gases is very small, would be readily attainable by using this temperature responsive MAMS. Ma et al. [40] has successfully demonstrated a linear relationship between the mesh gate opening (D) and the temperature (T); $\mathrm{D}=\mathrm{D}_{0}+\alpha \mathrm{T}$, where $\mathrm{D}_{0}$ refers to the mesh size at $0 \mathrm{~K}$ and $\alpha$ is a constant, to predict the best temperature for the separation of a gas mixture. $D_{0}$ and $\alpha$ are affected by applied tert-butyl group in MAMS. 
The above temperature responsive MAMS was constructed of weakly bonded sheets via van der Waals interactions with one-dimensional gated channels [40, 41]. The inter-sheets bonding interactions may not stay stable in harsh flue gas applications. Thus, Wriedt and co-workers [42] had proposed an improved design of MAMS by introducing strong coordination bonds between the layers. PCN-200 (PCN = porous coordination network) was used instead of the amphiphilic ligand, to fit exactly one $\mathrm{CO}_{2}$ molecule in a short interlayer distance of the elastic polar pocket of PCN-200. The developed high density PCN-200-ac is stable up to $218^{\circ} \mathrm{C}$ with high $\mathrm{CO}_{2}$ uptake capacity of $129 \mathrm{gL}^{-1}$, which meets the temperature requirements for physical sorbents in a flue gas separation process. More importantly, PCN-200-ac showed no loss in capture capacity even after running a series of thirty adsorption-desorption cycles at $313 \mathrm{~K}$. By alternating heating and cooling of PCN-200-ac between $423 \mathrm{~K}$ and $296 \mathrm{~K}$, respectively, $\mathrm{CO}_{2}$ loading capacity of $1.13 \mathrm{mmolg}^{-1}$ was realised by flowing pure $\mathrm{CO}_{2}$ over the sample and $0.65 \mathrm{mmolg}^{-1}$ in $\mathrm{CO}_{2} / \mathrm{N}_{2}$ (15:85) mixture gas as flue gas simulant. This result emphasises the capabilities of the thermal-responsive PCN-200 as a sorbent to remove $\mathrm{CO}_{2}$ from flue gas [42].

Stimuli-responsive materials for gas separations commonly utilise materials sensitive to temperature, as shown in the examples given above, yet another stimulus may prove to be a more practical choice. In particular, photo-responsive materials for gas separation is highly appealing, the most popular amongst the reported external stimuli (redox, temperature, $\mathrm{pH}$, light, magnetic field etc.) without any by-product generation [37]. Photo-irradiation that brings about reversible changes in physicochemical properties generally works by isomerisation of the photo-chromic groups integrated in the bulk or surface of a membrane, where the membrane separation selectivity and regeneration efficiency can be tuned upon light irradiation [15, 20]. Generally, photochemical reactions are induced by ultraviolet, visible and infrared radiation, where molecules absorb the light energy through excitation of electrons. Efficiency of this process depends on the structure transformations of the photo-chromic groups (Fig. 1) as well as the energy of the light absorbed. Light absorption causes the molecules to become excited, thus initiating various photo-chemical reactions [20]. The changes in photo-chromic groups' structures cause the changes in macroscopic properties of a membrane, including the charge, wettability and mass transport properties [15].

Park and co-workers [37] have demonstrated an instant $\mathrm{CO}_{2}$ capture and release platform by introducing an azobenzene (AZB) functional group to the organic linker of an MOF. Before UV exposure, the freshly made MOF sample manifested significant $\mathrm{CO}_{2}$ uptake of $22.9 \mathrm{~cm}^{3} / \mathrm{g}$ at room temperature and $1 \mathrm{bar}$, with a negligible adsorbed amount of $\mathrm{N}_{2}$. Up to $53.9 \%$ adsorbed $\mathrm{CO}_{2}$ can be released after $5 \mathrm{~h}$ exposure to UV irradiation as a result of the isomerisation of the AZB groups inside the pores of the MOF. The MOF adsorbent was able to return to its original state upon gentle heating at $60^{\circ} \mathrm{C}$ for $30 \mathrm{~min}$ in the dark. Potentially, the findings showed a possible efficient separation of gas molecules (uptake of $\mathrm{CO}_{2}$ and negligible adsorption of $\mathrm{N}_{2}$ ) with fast regeneration ability (release of $\mathrm{CO}_{2}$ to return to its original state through heating process) [37]. The working mechanism of photo-responsive materials, as well as the use of AZB and MOF in gas separation systems will be discussed in more detail and depth in later sections.

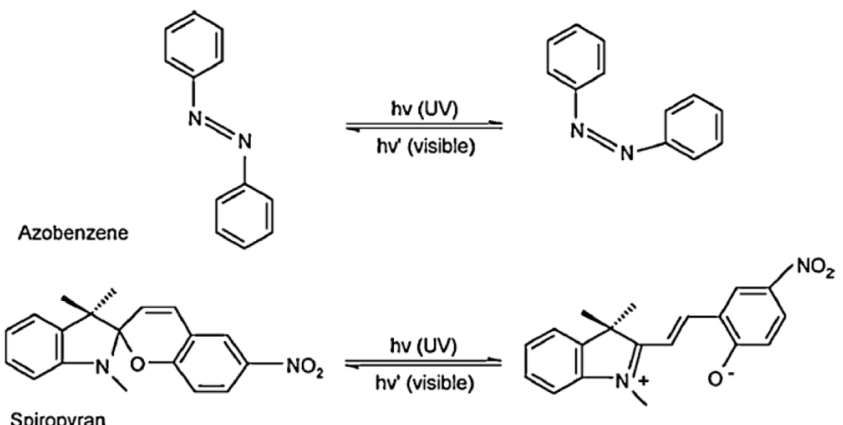

Spiropyran

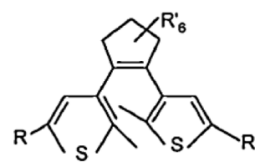
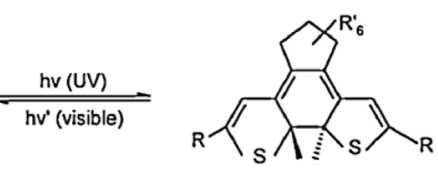

Diarylethene

Fig. 1. Examples of photo-chromic conjugates used in photo-responsive materials for gas separation; azobenzene, spiro-benzopyran and diarylethene, with their reversible photo-reactions. Reproduced with permission from Wandera et al. [15]; Copyright (2010) Elsevier and He et al. [20]; Copyright (2008) Elsevier.

Release triggers for adsorbed gases, such as thermal heating, tend to be very energy intensive [20], consuming a large amount of energy from the power plant. Despite this, most research works have used temperature as stimuli for the purpose of creating stimuli-responsive gas separation systems. Therefore, there is a strong motivation for the development of release triggers that do not require intensive energy from the plant, for example photo-responsive material triggered upon light irradiation, drawing from renewable energy resources such as solar energy. Unfortunately, utilisation of stimuli-responsive materials has not been focused on gas separations, especially with light as the stimuli. Next sections are focused on photo-isomerisation in polymer and integration of photo-responsive material in gas separation.

\section{Photo-Responsive Systems for Gas Separation}

Light is a very intriguing source of stimulus for a responsive trigger with the potential to drastically reduce energy costs if used with stimuli-responsive material that strongly absorbs light whilst desorbing adsorbed components [43-45]. In addition, stimulation by light is interesting as photo-excitation can be relayed in a very selective and speedy manner without imposing additional changes to the chemical potential of the system [36, 45, 46]. However, as photo-irradiation is an external stimulus, it requires the membrane housing to be optically transparent [15].

In a photo-reaction mechanism, two types of reactive entities can be distinguished, which are photo-sensitizers and photo-reactive molecules or moieties. Photo-sensitizers selectively absorb light and trigger a chemical reaction of another substance as a result. They function as "antennas" for light and from the excited state, transfer the absorbed energy through electron or radiative transfer [20]. Ding et al. [47] reported the usage of metal- 
lophthalocyanine based conjugated microporous polymers (MPc-CMPs, $\mathrm{M}=\mathrm{Co}, \mathrm{Ni}, \mathrm{Cu}, \mathrm{Zn}$ ) as photo-sensitizer to generate singlet oxygen $\left({ }^{1} \mathrm{O}_{2}\right)$. In the study, an oxygen-saturated solution of 1,3-diphenylisobenzofuran (DPBF) was irradiated with light in the presence MPc-CMPs to produce ${ }^{1} \mathrm{O}_{2}$, for application fields such as photodynamic therapy. Results demonstrated that at different photo-catalysis capability of MPC-CMPs, the central metal species $(\mathrm{M}=\mathrm{Co}, \mathrm{Ni}, \mathrm{Cu}, \mathrm{Zn})$ in the MPc will probably influence the triplet states, triggering the activation of photo-activity to generate ${ }^{1} \mathrm{O}_{2}$. In the report, $\mathrm{ZnPc}-\mathrm{CMP}$ and CuPc-CMP have demonstrated high efficiency for the generation of ${ }^{1} \mathrm{O}_{2}$ over other reported organic porous materials with lower energy required for light excitation.

As for gas separation, a novel strategy to utilise stimuli-responsive materials to trigger gas adsorption and desorption through the formation of radical triplets has been designed by An et al. [46]. In the work, MOF containing photo-reactive zwitterionic pyridinium 4-carboxylate units was exposed to UV light irradiation, forming radical triplets through intramolecular electron transfer. As this reversible light-responsive generation of radicals occurs at the major adsorption sites of the MOF, the $\mathrm{CO}_{2}$ adsorption could be controlled, where the light irradiation will cause the adsorption capacity of $\mathrm{CO}_{2}$ to decrease. Even though existing works are still quite scarce, it is evident that there is increasing interest for photo-responsive materials to be used for gas separation. In order to pursue the effort, a deeper understanding of the materials' properties, their interacting mechanisms with gas penetrants and integration approaches into the separation systems are of interest. More details of MOF in photo-responsive gas separation systems will be presented in the following sections.

\subsection{Photo-Reaction Mechanisms}

After absorption of light, photo-reactive molecules or moieties can undergo either irreversible or reversible reaction, which is typically known as isomerisation. Photo-isomerisation to one isomer and back is either both reversible photo-reaction processes, and one is a photo-chemical reaction and the other is a thermal reaction. These two reactions allow the photo-chromic unit to switch between two chromophoric moiety states [48], which brings about molecular changes such as group polarity, charge, and size [24, 49]. These molecular changes would show responses that can be visualized, that is, changes in macroscopic properties [15]. Fig. 2 shows an example of visual representation of these changes, which can affect the mass transport through the porous and non-porous barriers of a membrane separation layer.

Weh and co-workers [50] had produced a photo-responsive polymethacrylate membrane by chemically binding AZB which is capable of reversible photo-isomerisation (trans-to-cis states) to the side chain of polymethacrylate. Mass transport of gases thraough membranes is controlled by solubility and diffusivity, which depends on the relative size of the gas molecules and free volume within the polymer. When UV light was exposed to AZB, the spheroidic cis structure of AZB within the membrane matrix would fill the pores within the polymer, hence lowering the free volume of the membrane compared to when AZB is in the trans form. In the study, the cis-form of AZB hinders diffusivity more effectively compared to the trans isomer.

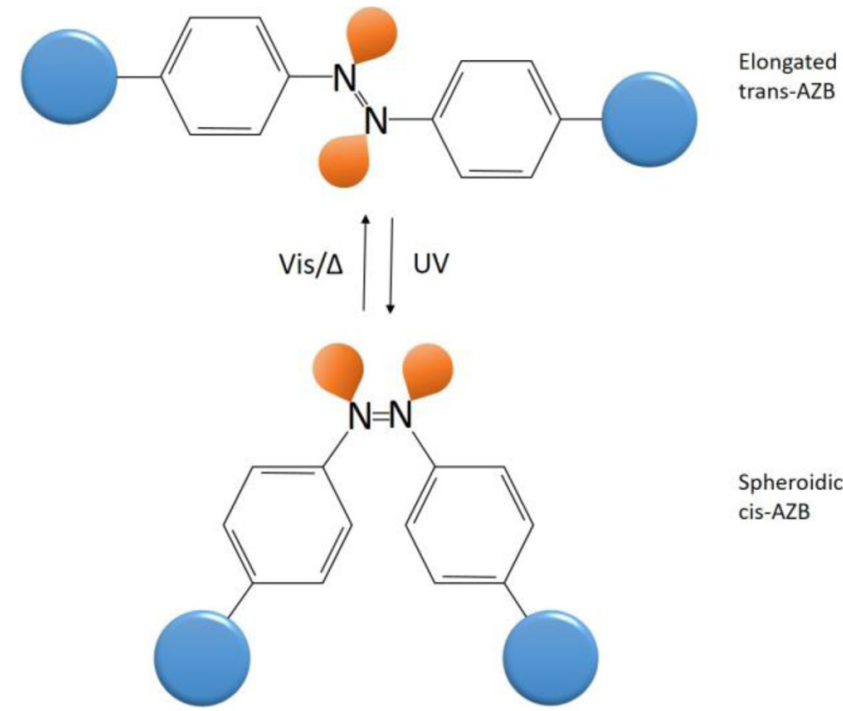

Fig. 2. Isomerisation of photo-chromic azobenzene (AZB) unit resulting in changes of conformation (size) and properties (polarity or charge) (Authors' own constructed figure).

In another work carried out by Weh et al [38], two zeolites were used to host AZB groups to control the gas permeation of a photo-responsive membrane. This intra-zeolitic photo-isomerisation was found to be capable of altering the molecular size, geometry and dipole moment of the AZB, resulting in changes of the vacant pore space for specific gas molecule to transport through the membrane. In their work, Weh et al [38] had summarized a few factors that contribute to the controlled permeation of gases. First, AZB isomers in the trans- and cis-forms can reversibly occupy different positions in the framework, which can present the AZB unit as a diffusion obstacle with different efficiencies depending on the position. Second, different configurations of the AZB isomers also means that the AZB unit can alter the free volume available in the membrane, depending on the space occupied by the isomers, to control the gas permeation and selectivity of a membrane [25, 50]. Third, the electrostatic interactions between AZB and gas penetrant could also play a role, especially between the polar cis-AZB and the more polar permeant gases. Dipole-dipole interactions between cis-AZB and polar gas molecules will increase the barrier to gas transport, in addition to the fact that AZB is bulkier in its cis-state compared to its trans-state [38].

However, there is another interpretation for MOFs containing photo-sensitive units affecting gas separation processes. Instead of undergoing photo-isomerisation from trans-to-cis state, light irradiation functions to increase the surface energy of the photo-sensitive MOF, thus, weakening the intermolecular interactions between adsorbed gas molecules and the MOF surface [44]. Although most azo-MOF reacts to UV irradiation by isomerisation, the same trigger caused structural oscillations due to the C-C-N bending movement of the azo-containing ligand instead [51]. Similarly, Lyndon et al. [45] found that the UV irradiation of an azo-containing ligand in MOF resulted in C-C-C and C-C-N bending movements due to the suppression of isomerisation. They observed that the bending modes resulted in a release of adsorbed $\mathrm{CO}_{2}$ molecules, due to 
the higher surface energy on the pore surface of MOF when irradiated. Indeed, this light-responsive functionality was used to trigger the reversible adsorption and release of $\mathrm{CO}_{2}$, finding that the exposure to UV light triggered an instantaneous release of up to $64 \%$ adsorbed $\mathrm{CO}_{2}$ with broadband radiation or concentrated solar sources.

While understanding the underlying mechanisms responsible for the observed behaviour is key to design the photo-stimulated $\mathrm{CO}_{2}$ capture and release process, studies addressing the issue are rarely reported because it is difficult to investigate the molecular-level mechanisms through experiments. Huang et al. [25] investigated the mechanism of $\mathrm{CO}_{2}$ capture and release using AZB functionalised MOF, calling into question the hypothesis that the drop in $\mathrm{CO}_{2}$ adsorption capacity is caused by the steric effects from the changes in pore morphology of the stimuli-responsive membrane, blocking the stronger metal oxide binding sites. Two MOF with AZB functional group attached to their ligands were investigated in their work. Even though isomerisation of the AZB groups to the cis-state reduced the pore diameter of the frameworks, they observed that the change in $\mathrm{CO}_{2}$ absorption capacity when photo-isomerisation occurred is actually caused by the alteration in local interactions from the accumulation of charge on metal oxides.

Through calculations of radial distribution functions between the $\mathrm{CO}_{2}$ centre of mass and two specific atoms of the framework in both trans- and cis-configurations of $\mathrm{AZB}$, it was suggested that the trans-AZB isomer provided extra interactions that led to the increased $\mathrm{CO}_{2}$ uptake [25]. While accessible surface area, free volume, pore morphology and adsorption sites can influence the uptake of $\mathrm{CO}_{2}$, adsorption enthalpy plays the largest role in controlling the capacity of $\mathrm{CO}_{2}$ adsorption in a porous structure. In the same work [25], it was found that the conventional $\mathrm{CO}_{2}$ binding sites were still available even when the AZB groups were in cis-state, not shielded as was suggested before. This further proves that the extra interactions between $\mathrm{CO}_{2}$ molecules and the MOF when AZB was in the trans-state was the main factor that led to the higher $\mathrm{CO}_{2}$ molecule adsorption capacity, as compared to when the AZB group was in the cis-state. This finding is in line with the $\mathrm{CO}_{2}$ binding energies when the AZB groups were in transand cis-configurations at -31.3 and $-27.5 \mathrm{~kJ} / \mathrm{mol}$, respectively.

Another photo-reaction process called photo-induced surface relief (PSR) formation is exhibited by azopolymer thin films, where surface modulation on the submicron scale occurs under light irradiation. Kameda et al. [52] found that the azopolymer in the trans-form supported by a base membrane were in the form of many localised particulate domains. However, after being exposed to irradiation, these particulate domains spread out to cover the surface of the base membrane. Based on the observation, they suggested that upon irradiation, the azopolymers were fluidised and went through plastic deformation during the relief-forming process, known as PSR, which is reversible. Gas permeability of a rubbery polymer is significantly higher than that of glassy polymer. The initially glassy azopolymer particulate domain, when irradiated, was plasticised and fluidised. Consequently, the change in state from glassy to rubbery could increase the gas permeability. Thus, the photo-responsive permeability of the membrane is caused by the thin layer of azopolymer spread on the surface of the base membrane. The photo-induced change in the azopolymer properties such as the increase in free volume was shown through the change in permeability under irradiation. Permeability of helium (He) gas increased by approximately $5 \%$ under irradiation with blue light, then returned to its initial value when irradiation was stopped.

While the PSR formation described above is very intriguing, other research suggested that the switchable properties of photo-responsive materials are derived from other causes. Most works explained that the changes in permeability or selectivity of the photo-responsive materials upon irradiation were due to the structure alteration when exposed to light. This morphology change could decrease the free volume of the material or membrane, hindering the gas diffusivity and thus becoming a diffusion obstacle. Another suggestion is that the polarity of the photo-chromic units is affected by isomerisation, consequently increasing the ability to attract polar gases. Yet a different proposed mechanism is that the irradiation of photo-responsive material could enhance its surface energy, which could trigger the release of adsorbed gases or increase its binding energy, thus enhancing the adsorption of target molecules. Either way, the effect of irradiation on photo-responsive systems depends on the properties of the photo-chromic molecules, which would be discussed in the next section.

\subsection{Photo-Chromic Units}

Based on the working mechanisms of photo-responsive materials in gas separation described in Section 3.1, this section focuses on the prevailing photo-responsive materials that have been researched for gas separation. Among the popular photo-chromic units used to design stimuli-responsive systems for the purpose of gas separation, AZB and diarylethene (DAE) are the most thoroughly researched. The changes in conformation of these photo-chromic units upon irradiation can be exploited to control the absorption properties or adsorption capacity of the microporous structures hosting these molecules [25, 53].

The planar trans-isomer of AZB has a greater distance between the aryl termini than the non-planar cis-isomer [54]. The distance between the para-carbon atoms in an AZB molecule in the trans-state is $9 \AA$, which changes to $5.5 \AA$ when isomerised into the cis-isomer $[35,37,55]$. This geometrical disparity between AZB isomers can be translated into bulk materials, altering their size. As for its reversible transformation capability, the thermodynamically stable AZB trans-isomer can be switched to the cis-isomer via irradiation with UV light $(\lambda=365 \mathrm{~nm})$, and is able to switch back to the trans-isomer through irradiation using visible light $(\lambda=455 \mathrm{~nm})$ $[36,56]$. Because the trans-AZB is thermodynamically more stable, the recovering of cis- to trans-isomer can also be done through thermal relaxation [53, 57]. In addition, the isomerisation also affects the polarity, dipole moment and required space, leading to a change in the sorption behaviour.

Through the reversible photo-stimulated changes of the photo-chromic units in a membrane, it can reversibly switch from a non-binding to a binding site for a target molecule by alternating photo-irradiation. The AZB isomers are significantly different in terms of their molecular geometries and electronic properties, rendering the feasibility of the AZB-containing membrane as a light-switchable $\mathrm{CO}_{2}$ valve for remote controllable gas permeation $[53,56]$. Such a photo-responsive function using AZB derivatives 
was demonstrated by Modrow et al. [57] through the incorporation of the derivatives into MOF. Irradiation with UV light to produce cis-isomers caused the $\mathrm{CH}_{4}$ adsorption capacity to increase, while reverting back to the trans-isomer by irradiation with visible light resulted in a reduction of the adsorbed $\mathrm{CH}_{4}$. Cis/trans isomerisation brought significant structural changes upon the host, leading to a substantial change in the sorption properties.

The MOF synthesised by Prasetya and Ladewig [39] containing AZB ligands showed photo-response towards $\mathrm{CO}_{2}$ adsorption in both static and dynamic conditions. Even though the AZB-containing $\mathrm{MOF}$ had demonstrated smaller surface area compared to the parent MOF, incorporation of the AZB units enhanced the affinity between the $\mathrm{CO}_{2}$ molecules and the MOF. This AZB-containing MOF was used as filler inside the Matrimid (MI) polymer and the polymer of intrinsic microporosity (PIM) to produce AZB-MOF/MI and AZB-MOF/PIM mixed matrix membranes (MMMs). Both the AZB-MOF/MI and AZB-MOF/PIM MMMs demonstrated significantly better permeability and $\mathrm{CO}_{2} / \mathrm{N}_{2}$ selectivity compared to their pristine polymers. It is worth noting that the performance of the AZB-MOF/PIM membrane had surpassed the 2008 Robeson Upper Bound.

Another photo-chromic unit that is very popular in the development of stimuli-responsive material is the DAE derivatives. When triggered by UV and visible light, DAE will undergo isomerisation involving ring-opening or ring-closing reactions due to their fatigue-resistant and thermally irreversible properties [24, 58, 59]. Both states typically show distinct absorption spectra that can be addressed by specific individual wavelength [49]. The reversible cyclisation and cyclo-reversion of DAE are enabled by a central $\Pi$-electron sextet. The ring-closing conformation after light-induced cyclisation allows an extended conjugation of the electrons due to the aromatic character of the thienyl rings. This leads to the higher electronic conductivity in a ring-closing state compared to the ring-opening state. DAE isomerisation brings about two decisive changes within the molecule; different electronic properties and a more rigid structure after cyclisation when the thienyl moieties become interconnected, thus reducing the mobility [21]. Moreover, DAE derivatives have very high photo-stability with over 104 photo-induced colouration/decolouration cycles and a lifetime exceeding a thousand years at $30^{\circ} \mathrm{C}$ [58]. These properties and characteristics make DAE derivatives attractive for use in low-energy gas separation by altering the separation properties such as the gas adsorption capacity, permeability and selectivity.

Fan and co-workers [24] attempted to overcome the reported trade-off associated with MOFs and membranes where high selectivity often comes with low adsorption capacity and vice versa. They approached the matter by using DAE units to demonstrate increased $\mathrm{C}_{2} \mathrm{H}_{2} / \mathrm{C}_{2} \mathrm{H}_{4}$ separation that can be switched with light as an external stimulus. A larger alteration in the DAE unit was required for the $\mathrm{C}_{2} \mathrm{H}_{4}$-loaded structure compared to the $\mathrm{C}_{2} \mathrm{H}_{2}$-loaded structure. Moreover, for the $\mathrm{C}_{2} \mathrm{H}_{2}$-loaded structure, other than the opening movement of the DAE unit, the whole DAE ligand was maintained, while an intense alteration on the entire DAE ligand would be necessary for $\mathrm{C}_{2} \mathrm{H}_{4}$. Such an intense overall alteration within the MOF skeleton would be completely rejected by the MOF due to its rigidity. The preferential adsorption of $\mathrm{C}_{2} \mathrm{H}_{2}$ over
$\mathrm{C}_{2} \mathrm{H}_{4}$ with a selectivity of 47.1 was also reflected in the isosteric heat of adsorption $\left(\mathrm{Q}_{\mathrm{st}}\right)$. The stronger host-guest interactions between $\mathrm{C}_{2} \mathrm{H}_{2}$ and the MOF were demonstrated by its larger $\mathrm{Q}_{\mathrm{st}}$ compared to $\mathrm{C}_{2} \mathrm{H}_{4}$.

From the above examples, it could be concluded that AZB in particular had garnered a lot of attention, in part due to its larger geometrical change compared to DAE. As steric effects could potentially affect the gas separation performance, a bigger change in the properties when isomerised could give a more pronounced change in performance. In addition, a study showed the performance of DAE-incorporated gas separation system decreasing after several cycles [21]. Further research needs to be done to understand the reason behind this so that measures can be taken to prevent or delay the occurrence. Another interesting avenue for future research is the integration of other photo-chromic molecules for gas separation, which is something that is severely lacking. However, the few works that have utilised other photo-chromic units to separate gases are given below.

Spiropyran is a photo-chromic unit that so far has rarely been used in gas separation. However, Healey et al. [60] had successfully incorporated spiropyran into MOF through a two-step post-synthesis modification of Zr-oxo nodes in MOF-808. Exposing spiropyran to UV irradiation would trigger an interconversion from the colourless spiropyran to its coloured merocyanine (MC) state [55, 61, 62]. The resulting MOF exhibited photo-sensitive response towards pore volume and $\mathrm{CO}_{2}$ uptake, due to the modified MOF having a markedly higher affinity towards $\mathrm{CO}_{2}$ than the parent MOF [60].

In another work carried out by Huang and co-workers [63], they had utilised another photo-sensitive unit, anthracene to design and synthesise covalent organic frameworks (COFs) to observe its ability to adsorb gas. Irradiation by light caused structural transformations that consequently resulted in changes of properties, including porosity, luminescence and r-electronic absorption. This thermally reversible reaction of the COF also showed a change in gas storage capability, showing potential for photo-responsive gas adsorption.

While research on AZB and DAE are relatively extensive, other photo-chromic units such as spiropyran and anthracene have not been studied much in comparison. This may due to limitations that is not clear as of yet. However, these very lacks of research done into other photo-chromic units makes these units more interesting, and give motivation to look into their prospects in gas separation systems.

\subsection{Techniques to Integrate Photo-Responsive Materials into the Gas Separation Systems}

MOF is already an attractive topic of research for gas storage and separation. More recently, it has been used to host photo-chromic units that could change properties as a response to external stimulus, which could then produce a change in gas separation performance. While photo-chromic guest molecules can be simply loaded or dangle from the MOF side chains, incorporating the molecules directly onto the backbone of the framework has the highest potential for drastic transformations, even though it is the most difficult to achieve. One of the common methods to integrate 


\section{Generation 1}
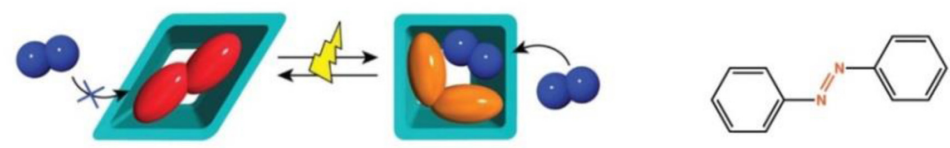

photoresponsive guest

Generation 2

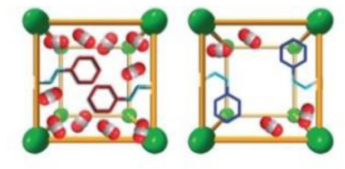

Generation 3

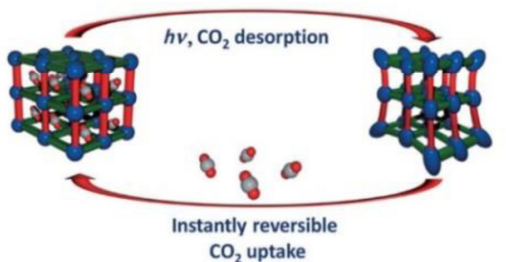

photoresponsive

linker side chain

Fig. 3. Integration of photo-responsive units into metal-organic frameworks. Reproduced with permission from Coudert [65]; Copyright (2012) American Chemical Society.

photo-responsive units is by incorporating them into MOF or membrane matrix, which can be classified into three generations [64, 65], as shown in Fig. 3. MOF is a class of microporous crystalline material comprised of metal centre and organic ligands, periodically joined to give a crystalline porous array [45]. It comprises of very large surface area as well as free volume with low density [66]. In terms of chemistry properties, MOF is chemically and thermally robust, in addition to having the ability to retain structural rigidity during reversible adsorption and desorption [22, 36]. Hence, it is a promising host for photo-chromic units, which sparked many investigations into energy-efficient gas capture and release that can be externally triggered [25].

The first generation of MOF is loaded with photo-responsive guest molecules, lending them an extrinsic photo-responsive property [32, 39]. However, this approach is not applicable in all cases as the isomerisation of the guest molecule can be blocked by the host framework [35, 50]. For instance, Hermann and co-workers [67] observed that the loading of AZB units into MIL-53(Al) has not only produced densely packed MOF with reduced pore volume, but also prevented AZB trans-to-cis isomerisation.

By depositing UiO-67 layer on $\alpha-\mathrm{Al}_{2} \mathrm{O}_{3}$ support, Knebel et al. [35] loaded AZB molecules into the matrix of UiO-67 membrane. Results showed that AZB had completely filled the pores of UiO-67, inhibiting isomerisation of AZB necessary for photo-switching. After in situ desorption of AZB was done to reduce the amount of AZB loaded inside the membrane, only then could gas permeation be observed. In this case, $\mathrm{H}_{2}$ and $\mathrm{CO}_{2}$ permeances decreased when the $\mathrm{AZB}$ units were in trans-state, with $\mathrm{CO}_{2}$ being more significantly affected due to its size. This observation was attributed to the gating mechanism where $\mathrm{AZB}$ in the trans-state reduced the aperture of the gate for gas molecules to diffuse through.

The second generation of MOFs differs from the first by having the photo-responsive side chains directly within the framework organic linkers [39]. Of the three generations of photo-responsive MOFs, this generation is the most researched, with researchers commonly linking the photo-chromic unit covalently to the organic linker. Several materials have shown the ability to allow the AZB side-chain to retain its photo-isomerisation ability [57], resulting in a change in pore dimensions when irradiated and subsequently altering its gas separation capabilities [37].

For example, Li and co-workers [68] investigated the capability of PCN-250 with AZB ligands as second generation MOF in light-induced swing adsorption (LISA) to capture and release $\mathrm{CO}_{2}$ using solar energy instead of electricity. The metal clusters of the MOF gave it great structural stability while the flexibility was provided by the AZB-containing ligands. Exposing the MOF to a UV light source would cause the ligand to undergo a suppressed bending in a non-periodic fashion which would release the adsorbed $\mathrm{CO}_{2}$ through the LISA process and regenerating the MOF. Intermittent light exposure demonstrated a reversible $\mathrm{CO}_{2}$ capture and release, or dynamic adsorption. An interesting observation was that reversing the cis-state AZB back to the trans-state AZB did not require exposure to visible light or heat treatment. The significantly accelerated relaxation of AZB-containing ligands in the MOF happened in only 5 minutes at room temperature in the dark. Reusability tests after five cycles showed only a 7.3\% and 4.9\% loss in adsorption and LISA capacity, respectively, confirming the robustness of the MOF and its promising function for stimuli-responsive capture and release applications.

In another work carried out by Luo et al. [58], they have constructed a porous diarylethene MOF (DMOF) using a DAE derivative with two $\mathrm{N}$-donor sites. $\mathrm{N}$ - and O-donor ligands were used to interact with dicarboxylate ligand of biphenyl-4,4'-dicarbocxylic acid ( $\mathrm{H}_{2}$ bpdc). The large skeletal structure provided by the photo-responsive DAE group allows for the construction of porous DMOFs. They found that this DMOF adsorbed $\mathrm{CO}_{2}$ selectively over $\mathrm{N}_{2}$ due 
to the quadruple moment of $\mathrm{CO}_{2}$. After UV irradiation, the uptake of $\mathrm{CO}_{2}$ increased significantly; 4 times the amount without UV irradiation.

While the third generation of photo-responsive MOFs is the most difficult to achieve, it is also the most promising of the three classes of MOFs. This generation of photo-responsive MOFs incorporates a photo-chromic unit directly in the "backbone" on the linker, not as a side chain [39, 69]. Using this method, the light stimuli will directly affect the framework itself, providing the potential to demonstrate the most dramatic transformations [65].

For an example, Tang et al. [70] had utilised AZB as the base in the backbones in conjunction with Lewis bases by covalently bonding them to design novel photo-switchable sorbents for $\mathrm{CO}_{2}$ capture. Results demonstrated that the difference in binding strength between the designed molecules and $\mathrm{CO}_{2}$ was hardly affected by electronic and steric effects. However, by substituting various groups into the ortho position with respect to the carbon atom of the benzene group of the azo moiety, $\mathrm{CO}_{2}$ molecules were expected to form intramolecular $\mathrm{H}$ bonds with the substitution groups. These bonds would then enlarge the difference of $\mathrm{CO}_{2}$ binding strengths of the cis- and trans-isomers so that the $\mathrm{CO}_{2}$-sorbent interaction can change from strong in one isomer to weak in the other by using light as stimulus, leading to a significant decrease in energy needed for sorbent regeneration.

DAE moieties had also been used as a building block for the construction of PIM by Becker et al. [21]. The properties of the DAE-PIM were studied during cyclisation and cyclo-reversion with light as the trigger. Porosity in PIMs is derived from the interstitial voids that are produced by the inefficient space packing of the polymer chains due to the building blocks possessing an inherent kink to their structure. Even though the geometrical change of $\mathrm{DAE}$ as a result of isomerisation is not as dramatic as AZB, DAE has a rigid and essentially planar structure in the ring-closing form and a slightly bent geometry in the ring-opening form. In this case, the stiff but kinked structure of PIMs is key to the microporosity of the polymer, as the inefficient chain packing determines the percolated free volume that is accessible for target adsorption molecules. Isomerisation of DAE altered the distribution of electrons, but when stored in the dark at room temperature, DAE-PIM did not revert to the ring-opening isomer even after weeks of storage. Thus, as long as not exposed to visible light, DAE-PIM would not return to the ring-opening isomer for a long time. As for the separation performances, making a composite DAE-PIM with MI, yielding DAE-PIM@MI increased the diffusion coefficient (D) for both $\mathrm{O}_{2}$ and $\mathrm{CO}_{2}$ by $10 \%$. Most importantly, the isomerisation of the composite significantly reduced $\mathrm{D}$ value in the ring-closing state while cyclo-reversion into the ring-opening state enhanced the D value. Even though cycling between the ring-opening and ring-closing states was reversible through photo-induced switching, however, after five cycles, the intensity of the band at $610 \mathrm{~nm}$ only retained $80 \%$ of the original value. This might due to the degradation of DAE moieties, hence preventing more comprehensive cyclo-reversion [21].

Porous coordination polymers (PCP) also have highly regular nanopores useful for storage and separation as well as being responsive to guest molecules such as photo-chromic groups while retaining high regularity. In a work demonstrated by Yani et al
[32], AZB was incorporated into a flexible host compound, $\left[\mathrm{Zn}_{2} \text { (terephthalate }\right)_{2}$ (triethylenediamine) $]_{\mathrm{n}}$ whose pores become deformed due to the incorporation of the aromatic guest molecules. UV irradiation triggered a partial expansion of the host framework when AZB was isomerised from trans- into cis-form. The composite did not initially adsorb $\mathrm{N}_{2}$, possibly due to the pore blockage of the host by AZB, but upon UV irradiation, the adsorption amount increased by 8.3 fold. The significant increment of the adsorption capability of the composite is attributed to the large structural change of the host framework that was triggered by the isomerisation of AZB, as the tetragonal form in cis-AZB expanded the host framework to allow diffusion of gas molecules into the pores.

While a lot of research on photo-chromic materials for the purpose of light-triggered gas separation used MOF as the host, a considerable amount of work has also been done on introducing this switchable function into polymers and membranes for gas separation. Ulbricht [71] stated that even though strides have been made in the development of microporous inorganic membranes, subtly fine-tuning the properties of the barrier, a necessity for molecule-selective separations, is only possible using polymeric structures. Membrane processes for gas separation has been extensively researched, but they have fixed permeation properties. In gas adsorption processes, regeneration of the adsorbent is usually costly and energy intensive [43]. Therefore, interest in producing membranes with switchable function to develop a multifunctional membrane has been growing. A change in the photo-responsive guest molecule by external stimuli would simultaneously transform the membrane according to the alteration of the guest molecules [32].

For example, Weh et al. [50] attempted to prepare photo-responsive membranes using two methods; physically loading AZB in poly(methylmethacrylate) (PMMA) matrix and by chemically binding AZB to the side chain. For the membrane with AZB chemically bound to its side chain (PMAZB), the flux rates of gases permeating through the membrane appeared in decreasing order of $\mathrm{H}_{2}>\mathrm{n}-\mathrm{C}_{4} \mathrm{H}_{10}>\mathrm{CH}_{4}>\mathrm{SF}_{6}$. They noted that the permeability of gases in the cis-state of the membrane was lower compared to the trans-state, due to the spheroidic cis-AZB being a more effective diffusion obstacle compared to the elongated trans-AZB. On the other hand, the permeability of PMMA membrane with AZB physically dissolved in the membrane matrix has shown insignificant influence when switching in between the trans- and cis-states. The flux rates of gases for this membrane (physically loading AZB in PMMA matrix) is significantly lower compared to the pure PMMA membrane and the PMAZB. Authors concluded that the physically loaded AZB in PMMA matrix caused $\mathrm{AZB}$ to occupy the free volume in the membrane, hence reducing the transport capacity of the membrane drastically as a result. In addition, the membrane in cis-state had a higher dipole moment compared to the membrane in trans-state, leading to the high permeation rate for a polar permeate compared to the non-polar gases [24, 38].

Another type of membrane called surface-mounted MOFs (SURMOFs) were fabricated by growing MOF thin films continuously in layers to obtain monolithic, crystalline MOF films, as reported by Wang and co-workers [72]. The fabricated membranes have a pillared layer structure of $\mathrm{Cu}_{2}(\mathrm{AzoBPDC})_{2}(\mathrm{AzoBiPyB})$ con- 

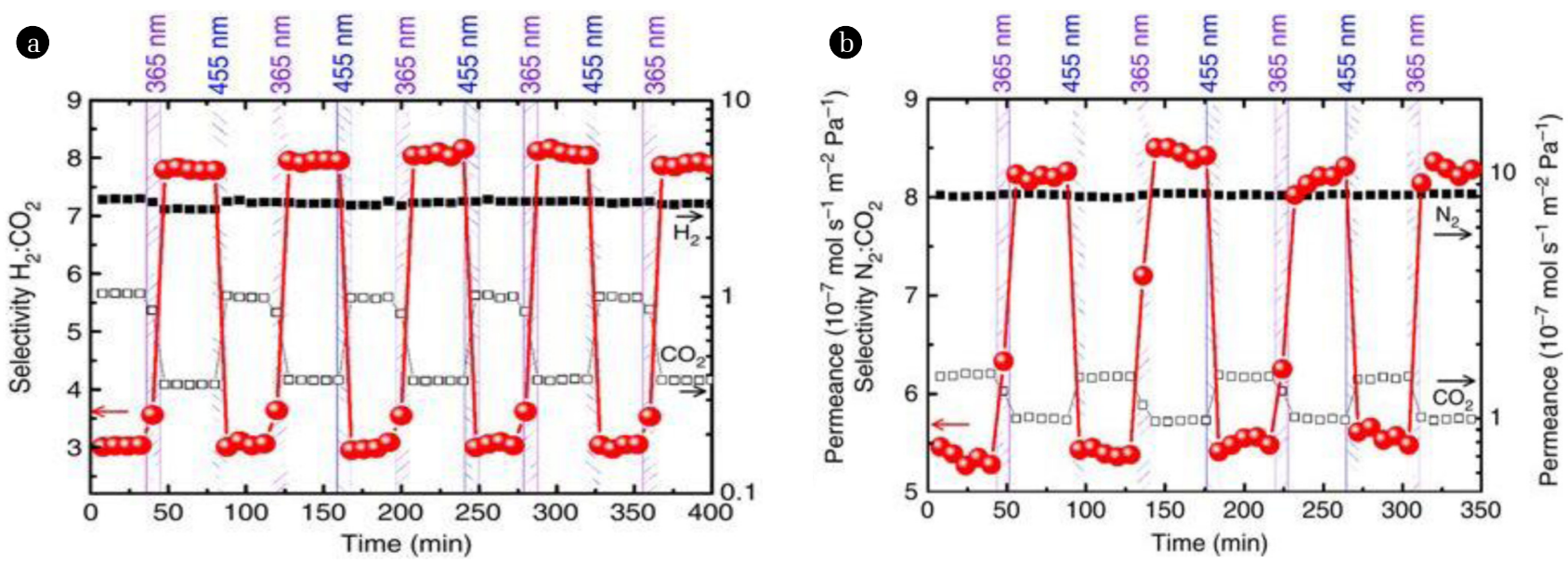

Fig. 4. The separation of (a) $\mathrm{H}_{2}: \mathrm{CO}_{2}$ and (b) $\mathrm{N}_{2}: \mathrm{CO}_{2}$ gas mixtures, respectively through the membrane under dynamic photo-switching. Red spheres with the scale on the left-hand side represent the separation factors. (a) $\mathrm{H}_{2}$ and $\mathrm{CO}_{2}$ as well as (b) $\mathrm{N}_{2}$ and $\mathrm{CO}_{2}$ permeances are represented by black solid squares and black open squares, respectively, and the logarithmic scale is shown on the right-hand side. Reproduced with permission from Wang et al. [72].

taining AZB on asymmetric mesoporous aluminium oxide support. Switching the AZB-groups in the membrane from trans- to cis-state only reduced the $\mathrm{H}_{2}$ permeance slightly while the $\mathrm{CO}_{2}$ permeability was significantly affected. Fig. 4 shows the reversible photo-switching of permeability and separation factors of $\mathrm{H}_{2}: \mathrm{CO}_{2}$ and $\mathrm{N}_{2}: \mathrm{CO}_{2}$ mixtures through the membrane. The cis-AZB with higher polarity had a stronger attraction with the fairly strong quadruple-moment of $\mathrm{CO}_{2}$, resulting in a decrease in the $\mathrm{CO}_{2}$ diffusion rate. On the other hand, the interaction between $\mathrm{H}_{2}$ and the membrane was not affected by the switching of isomers. This led to an increase of trans/cis permselectivity from 3.0 in the trans-state to approximately 8.0 in the cis-state of the membrane.

In the same work [72], the separation switching of $\mathrm{N}_{2}: \mathrm{CO}_{2}$ mixture was found to be reversible as well, with a selectivity increase from 5.5 to 8.5 when the AZB was switched from trans-state to cis-state, as $\mathrm{N}_{2}$ permeability was hardly affected by the trans-to-cis switching. Amazingly, not only the separation factor of the photo-responsive membrane can be switched between a minimal and a maximal values, but any values between the aforementioned ranges can be achieved by adjusting the irradiation times or by manipulating the relative intensities of the multi-component light irradiation. Therefore, the separation factor of the permeate gases can be continuously tuned. One of the advantages in being able to tune the separation factor of the membrane can be demonstrated by the ability to control the flammability and the safety of the pre-combustion mixture of $\mathrm{H}_{2}: \mathrm{CO}_{2}$ in the presence of air or oxygen. Using this system, each composition in between the ranges can be tuned so that the hazardous or non-hazardous condition of the gas mixture can be controlled remotely [72].

Other than MOF and polymeric membranes, COFs, a new class of crystalline porous polymers have also been constructed with AZB as part of their building block. Having permanent porosity, structural periodicity and thermal stability, COFs have been garnering interest recently, especially for gas absorption and storage owing to their large pore volume, high surface area and low density [63]. As COFs are constructed through atomically precise integration of building blocks, the variations of pore sizes, functions and structures of COFs are dependent on the diversity of the functional building blocks [69]. In fact, numerous groups reported the excellent gas pair separation using two-dimensional layered-stacking COF-COF composite membrane to separate $\mathrm{H}_{2} / \mathrm{CO}_{2}, \mathrm{H}_{2} / \mathrm{N}_{2}, \mathrm{H}_{2} / \mathrm{CH}_{4}$ [73], and $\mathrm{CO}_{2} / \mathrm{N}_{2}[74,75]$ gas mixtures, together with an enhancement in permeability to surpasses the Robeson upper bounds. By integrating photo-responsive AZB to control the conformational changes of COF composite membrane, a more precise gas pair separation is expected [63].

Zhu and Zhang [54] utilised a porous organic polymer (POP) to investigate whether the change in AZB polarity would be adequate to change the bulk material properties. POP is a porous material that is lightweight with intrinsic porosity, excellent physiochemical stability as well as structural tenability. It was observed that a bulky substituent on the AZB moiety hindered the photo-responsiveness of the frameworks due to the steric hindrance that suppressed the isomerisation. These polymers demonstrated reversible changes in pore size distribution and $\mathrm{CO}_{2}$ uptake using UV light irradiation and thermal treatment. Even after several cycles, the absorption properties were similar, indicating robustness and good switchability of the pore structures of the POPs. While photo-responsive MOFs with AZB units could induce the release of $\mathrm{CO}_{2}$ by isomerisation from trans-to-cis, the POPs showed a unique inverse alteration by absorbing more $\mathrm{CO}_{2}$ upon trans-to-cis isomerisation. This is because the dipole-quadrupole interaction between polar $\mathrm{CO}_{2}$ molecules and more polar cis-AZB was enhanced.

So far, the integration strategies of photo-chromic units into MOFs and membranes are similar. Most photo-chromic research for gas separation has focused on the second generation incorporation method. Even though the results of the first and second generation methods have shown promise, researchers have started to venture into the third generation of photo-chromic integration, which, while difficult, has the best chances of showing prominent results. 


\section{Practicability of Photo-Responsive Membranes for Gas Separation}

Membrane adsorbers for gas separation have vital advantages compared to conventional porous adsorbers due to the membrane pore structure which allows directional convective flow through the pores, greatly reducing the distance for pore diffusion. In membrane adsorption, separation occurs based on the reversible binding of molecules on the functionalised pore walls. Therefore, development of membrane adsorbers needs to progress towards optimising the pore structure as well as surface layer functionality so that a maximum number of binding sites with optimum accessibility can be obtained [71]. Conventional methods to regenerate adsorbents such as vacuum and heating are not energy efficient [66], providing incentive towards research on photo-responsive adsorbents that can be regenerated using less energy at a reduced cost [51]. Various polymers and membranes have also been investigated for incorporation of photo-chromic units to manipulate gas separation performance $[21,39]$. Utilising a photo-responsive membrane could open possibilities for switchable, multi-functional membranes, in addition to potentially reducing gas separation costs. While many attributed the change in gas separation performance to the change of the conformation of photo-chromic groups, some also mentioned that the changes were brought by the alteration of dipole moment of the photo-chromic groups and their interaction with target molecules upon isomerisation [24].

Park et al. [37] chose to synthesise MOF with AZB as a dangling group as $\mathrm{CO}_{2}$ adsorption on MOF at room temperature is relatively high. There was a delay in the change in gas adsorption isotherms when exposed to UV irradiation due to the slow isomerisation in the PCN-123 MOF caused by the steric hindrance among AZB groups. Initially, the AZB groups were predominantly in the trans-state, which is a relatively stable state. Upon being exposed to UV irradiation, only some of the AZB groups changed into cis-state right away, causing a decrease of $\mathrm{CO}_{2}$ uptake by $26.6 \%$, while more trans-AZB later became cis-AZB as they were triggered by the initial conformational changes, leading to the gradual reduction of gas uptake. The overall reduction of $\mathrm{CO}_{2}$ uptake after irradiation was $53.9 \%$. The change in position of the dangling benzene group in PCN-123 caused the reduction in gas adsorption capacity. The electron density of the cis-isomer around the $\mathrm{Zn}_{4} \mathrm{O}$ cluster is higher than the trans-isomer, making it harder for $\mathrm{CO}_{2}$ molecules to approach and occupy the metal clusters in the cis-state, which are the main adsorption sites for $\mathrm{CO}_{2}$ molecules.

PCN-123 was used in addition to another MOF, $\mathrm{Cu}_{2}(\mathrm{AzoBPDC})_{2}(\mathrm{AzoBiPyB})$ by Yang et al. [43] as photo-responsive adsorbents for $\mathrm{CO}_{2}$ adsorption. Similar to Park et al [37], they arrived to the conclusion that the decreased uptake of $\mathrm{CO}_{2}$ for both AZB-containing MOFs upon trans-to-cis isomerisation due to irradiation was attributed to the blocking of metal nodes of the MOF by cis-AZB, which function as the adsorbing sites. As both MOFs operate under the same mechanism, they hypothesised that this mechanism would apply for materials with exposed metal centre.
Another MOF combining AZB and acrylamide ligands was synthesised and reported by Dang and co-workers [66]. Results showed excellent selectivity for $\mathrm{CO}_{2}$ adsorption over $\mathrm{CH}_{4}, \mathrm{O}_{2}$, $\mathrm{CO}$ and $\mathrm{N}_{2}$ gasses. Exposure to UV light caused the AZB in the MOF to isomerise rapidly from trans- to cis-state, subsequently reducing the size of the pore accommodating adsorbed $\mathrm{CO}_{2}$ to incite $\mathrm{CO}_{2}$ release, thus regenerating the adsorbent. Dynamic irradiation caused instantaneous $\mathrm{CO}_{2}$ release with $21 \%$ desorption capacity, showing promise for a low energy $\mathrm{CO}_{2}$ release in adsorption process.

A concern with using UV light to trigger trans-to-cis photo-isomerisation of $\mathrm{AZB}$ is that the UV light can destroy and reduce the lifespan of materials and hinder applications. To counter the problem, advanced AZB derivatives that can be reversibly switched without UV irradiation were developed [76]. AZB is particularly popular in the development of visible-light-activated photo-responsive materials as it can be tailored to demonstrate complete addressability in the visible region. Castellanos et al. [48] fabricated two MOFs; aluminium-based ML-53(Al) and zirconium-based $\mathrm{UiO66}(\mathrm{Zr})$ that were responsive to visible light using the same ortho-fluoroazobenzene linker with different inorganic secondary building blocks (SBUs) to observe the impact of the framework structure.

Both MOFs have pore dimensions that can accommodate the AZB group within the large voids in the crystalline network in a disordered orientation. The AZB units forced the MIL-53(Al) lattice into the form with large pores, as the benzene rings of the terephthalate linkers allow a certain rotational degree of freedom, opening to the possibility of the bulky AZB units to cause rotation of the terephthalate rings away from parallel orientation. It is suggested that the isomerisation efficiency of AZB in F-azo-MLL-53(Al) was drastically reduced due to congestion of AZB in the MOF. On the other hand, F-azo-UiO-66(Zr) responded readily to both green and blue light, with green light triggering isomerisation much more efficiently compared to UV radiation [48].

In situ photo-irradiation experiments where samples were irradiated during $\mathrm{CO}_{2}$ adsorption showed that for both frameworks, the pure MOFs without AZB moieties could adsorb more $\mathrm{CO}_{2}$ compared to their photo-responsive counterparts as the pores were partially occupied by the pendant AZB moieties. Even though F-azo-UiO-66(Zr) showed good photo-chromic response under visible light, the $\mathrm{CO}_{2}$ adsorption capability was not affected by green light, concluding that the geometrical alteration of the side group of the linker in this MOF had no influence on the total volume of $\mathrm{CO}_{2}$ molecules that can be hosted within the pores. Conversely, the $\mathrm{CO}_{2}$ adsorption capability of F-azo-ML-53(Al) dropped by $10 \%$ during exposure to green light due to the hindered isomerisation events. As AZB is not fluorescent, the excess of light energy that was absorbed by the photo-chromic units must dissipate via radiationless photo-physical deactivation, where the electronically excited azo moieties relaxed through intramolecular vibrations that were transferred to the framework, thus increasing the surface energy and locally producing heat. It was measured that the decrease of $10 \%$ in the $\mathrm{CO}_{2}$ adsorption ability was comparable to that produced by an increase in overall temperature by $10^{\circ} \mathrm{C}$. Although hindered isomerisation is typically undesirable in photo-chromic 
applications, in this case it led to the change in $\mathrm{CO}_{2}$ adsorption capacity [48].

Both AZB and DAE units were used to synthesise MOF by Fan et al. [51] to observe adsorption of $\mathrm{CO}_{2}, \mathrm{C}_{2} \mathrm{H}_{2}$ and $\mathrm{C}_{2} \mathrm{H}_{4}$ gases. Exposure of the MOF to UV light resulted in C-C-N bending of the AZB-containing ligand rather than trans-to cis-isomerisation [45, 68], while UV irradiation at $320 \mathrm{~nm}$ exhibited partial transformation of DAE units from ring-opening to ring-closing form. $\mathrm{CO}_{2}$ adsorption occurred closer to the photo-sensitive centre of the MOF, leading to a larger effect on $\mathrm{CO}_{2}$ adsorption upon UV irradiation compared to $\mathrm{C}_{2} \mathrm{H}_{2}$ and $\mathrm{C}_{2} \mathrm{H}_{4}$ adsorption. This gave the MOF ability to alter the selectivity of the gas mixtures as well as adsorption capacity [51].

While light is an attractive trigger for gas capture and release, $\mathrm{Li}$ et al. [64] raised the issue of high volume of materials needed for light to pass in a large scale operation. Therefore, they developed a magnetic induced swing adsorption (MISA) strategy by incorporating magnetic nanoparticles into the MOF to generate heat when exposed to magnetic field. This field can fully penetrate the MOF, unlike light to release the adsorbed gas from MOF. MISA process, which requires electricity is combined with LISA process obtain a low-energy and efficient approach for gas adsorption. Integrating $\mathrm{Fe}_{3} \mathrm{O}_{4}$ magnetic nanoparticles into PCN-250 MOF that contains AZB linkers produced a dual stimuli-responsive MOF that can enhance the gas release up to $96.8 \%$ of $\mathrm{CO}_{2}$ desorption.

In an absorption process through a dense polymeric membrane, the effective flux rate represents the net transport through the membrane. This is governed by both the diffusivity and solubility below the glass transition temperature of the polymer [50]. By incorporating photo-chromic molecules inside the absorption system, the isomerisation state of the photo-chromic groups can be manipulated by light, consequently providing switching control over the flux and the separation factor of the gas permeating through the membrane. Therefore, the permeability of gases as well as the separation factor or perm selectivity can be continuously and remotely tuned by adjusting the ratio of trans- and cis-isomers of the photo-chromic groups [72].

Most works investigating photo-responsive materials necessitates the use of UV light to activate the photo-sensitive moieties, which comes with several disadvantages such as poor selectivity, low penetration into most media and potentially damaging. Therefore some research has been done to utilise visible light as a trigger instead, which can be beneficial especially in the aspect of exploiting solar energy as visible light is predominant in the solar spectrum [48]. Müller et al. [76] used o-fluoroazobenzene moieties to functionalise a nanoporous, photo-switchable MOF thin film that can undergo isomerisation with visible light. Another advantage to avoiding UV light is that the ligand-to-metal-charge transfer, which is excited by UV light in Cu-paddle-wheel MOF structures would not be stimulated using visible light. Therefore, very high isomerisation yields of AZB groups can be achieved, which is unprecedented in MOFs. Green and violet light were used to control the isomerisation state of the photo-chromic groups, consequently controlling the gas uptake and separation factors of $\mathrm{H}_{2}: \mathrm{C}_{2} \mathrm{H}_{4}$ and $\mathrm{H}_{2}: \mathrm{C}_{3} \mathrm{H}_{6}$ mixtures through MOF membranes.
In the same work [76], it was found that $\mathrm{H}_{2}$ permeability through a membrane of thin SURMOF film grown on a mesoporous $\mathrm{Al}_{2} \mathrm{O}_{3}$ substrate was hardly affected by the trans-to-cis switching. However, the permeability of $\mathrm{C}_{2} \mathrm{H}_{4}$ and $\mathrm{C}_{3} \mathrm{H}_{6}$ increased by approximately 25 and $30 \%$, respectively when the membrane was irradiated with green light. The original permeance and selectivity could be recovered by irradiating with violet light. The $\mathrm{H}_{2}: \mathrm{C}_{2} \mathrm{H}_{4}$ selectivity can be switched between 6.6 and 8.2 while the $\mathrm{C}_{3} \mathrm{H}_{6}$ selectivity can be switched between 8.8 and 12.6. Tuning these selectivities between the aforementioned ranges can be done continuously by adjusting the irradiation times or by mixing the green and violet light.

As the separation of $\mathrm{H}_{2}$ and $\mathrm{CO}_{2}$ showed no significant changes upon isomerisation and the polarity difference between trans- and cis-AZB was unable to influence the adsorption of the apolar hydrocarbons, the conclusion was that the separation by the photo-responsive $\mathrm{Cu}_{2}\left(\mathrm{~F}_{2} \text { AzoBDC }\right)_{2}$ (dabco) membrane was driven by steric effects instead of changes in the dipole moment. In the trans-state, the SURMOF had smaller pore diameter, resulting in a lower hydrocarbon permeability compared to the cis-state. This finding is in line with the larger switching impact observed for the larger hydrocarbon molecules while the permeability of the smaller $\mathrm{H}_{2}$ molecules was not affected. It was believed that the steric effect superimposed the polarity switching effect of AZB, leading to no switching impact on the $\mathrm{H}_{2}: \mathrm{CO}_{2}$ separation [76].

MMMs incorporating light responsive MOFs into MI polymer matrix were synthesised by Prasetya et al. [77] to observe the light-responsive gas separation. JUC-62 and PCN-250 are both generation-3 light responsive MOFs with significantly reduced $\mathrm{CO}_{2}$ adsorption capacity when exposed to UV light. On the other hand, the permeation of $\mathrm{N}_{2}$ hardly changed with photoswitching. A maximum switchability of $8.5 \%$ was obtained using $15 \mathrm{wt} \%$ JUC-62-MI, which was lower than expected. This was caused by the rigid structure of MI polymer matrix that limited the ligand bending of the photo-responsive MOFs. While PCN-250-MI had higher adsorption capacity than JUC-62-MI as PCN-250 had more active sites for $\mathrm{CO}_{2}$ permeation, both $\mathrm{MMMs}$ had superior $\mathrm{CO}_{2} / \mathrm{N}_{2}$ ideal selectivity compared to pristine MI membranes. This was due to the good interaction and dispersion of MOFs inside the polymer matrix as well as the good affinity between the light-responsive AZB units in the MOFs with $\mathrm{CO}_{2}[24,54]$. Long-term performance showed negligible changes for 15 wt\% JUC-62-MI and a slight decrease in 10wt\% PCN-250-MI, thus showing promise for the construction of photo-responsive anti-ageing material for $\mathrm{CO}_{2} / \mathrm{N}_{2}$ separation.

In adsorption, the change in position of the photo-chromic dangling group can occupy or block the adsorption sites, which would reduce the adsorption capacity. On the other hand, the conformation change of the photo-chromic units can alter their efficiency as diffusion obstacles in absorption by changing the free volume. The polarity of the photo-chromic isomer is another factor that can affect both the permeability in absorption and adsorption capacity when polar target molecules are involved. There are many possibilities for photo-responsive materials in the gas separation industry, not only to tune the separation factor remotely to change the permeability and gas adsorption capacity. For instance, the 
Table 1. Research on Photo-sensitive Materials for Gas Separation to Date

\begin{tabular}{|c|c|c|c|c|}
\hline Photo-chromic moiety & Process & Investigated gas & Host for the photo-chromic units & Ref. \\
\hline AZB & Adsorption & $\mathrm{CO}_{2}$ & $\mathrm{MOF}$ & {$[25,37,43,45,48,68]$} \\
\hline AZB & Adsorption & $\mathrm{CO}_{2}$ & Dual stimuli-responsive MOF & [64] \\
\hline $\mathrm{AZB}$ & Adsorption & $\mathrm{CH}_{4}$ & $\mathrm{MOF}$ & [57] \\
\hline AZB & Adsorption & $\mathrm{CO}_{2}, \mathrm{CH}_{4}, \mathrm{O}_{2}, \mathrm{CO}, \mathrm{N}_{2}$ & $\mathrm{MOF}$ & [66] \\
\hline AZB & Adsorption & $\mathrm{CO}_{2}$ & Lewis bases & [70] \\
\hline AZB & Adsorption & $\mathrm{N}_{2}$ & PCP & [32] \\
\hline AZB & Adsorption, absorption & $\mathrm{CO}_{2}, \mathrm{~N}_{2}$ & MOF, MI membrane, PIM & {$[39,77]$} \\
\hline AZB & Absorption & $\mathrm{H}_{2}, \mathrm{CO}_{2}, \mathrm{~N}_{2}, \mathrm{CH}_{4}, \mathrm{C}_{2} \mathrm{H}_{6}, \mathrm{C}_{3} \mathrm{H}_{8}$ & $\mathrm{MOF}$ & [35] \\
\hline AZB & Absorption & $\mathrm{H}_{2}, \mathrm{n}-\mathrm{C}_{4} \mathrm{H}_{10}, \mathrm{CH}_{4}, \mathrm{SF}_{6}, \mathrm{CH}_{3} \mathrm{OH}$ & PMMA membrane & {$[50]$} \\
\hline $\mathrm{AZB}$ & Absorption & $\mathrm{CH}_{4}, \mathrm{CO}_{2}, \mathrm{~N}_{2}$ & Zeolite-AZB membranes & {$[38]$} \\
\hline $\mathrm{AZB}$ & Absorption & $\mathrm{He}$ & Azopolymer membrane & {$[52]$} \\
\hline AZB & Absorption & $\mathrm{CO}_{2}$ & $\mathrm{POP}$ & [54] \\
\hline AZB & Absorption & $\mathrm{H}_{2}, \mathrm{CO}_{2}$ & SURMOF & [72] \\
\hline AZB & Absorption & $\mathrm{H}_{2}, \mathrm{C}_{2} \mathrm{H}_{4}, \mathrm{C}_{3} \mathrm{H}_{6}$ & SURMOF & [76] \\
\hline AZB, DAE & Adsorption & $\mathrm{CO}_{2}, \mathrm{C}_{2} \mathrm{H}_{2}, \mathrm{C}_{2} \mathrm{H}_{4}$ & MOF & [51] \\
\hline DAE & Adsorption & $\mathrm{CO}_{2}, \mathrm{~N}_{2}$ & $\mathrm{MOF}$ & [58] \\
\hline DAE & Adsorption & $\mathrm{C}_{2} \mathrm{H}_{2}, \mathrm{C}_{2} \mathrm{H}_{4}$ & $\mathrm{MOF}$ & [24] \\
\hline $\mathrm{DAE}$ & Absorption & $\mathrm{N}_{2}$ & PIM & [21] \\
\hline Spiropyran & Adsorption & $\mathrm{CO}_{2}$ & $\mathrm{MOF}$ & [60] \\
\hline
\end{tabular}

triggering using external stimuli can be used to manipulate the selectivity of gas mixtures to control the flammability of the mixture and thus the safety of the system. A summary of the research available on photo-sensitive materials for gas separation so far is listed in Table 1 and the basic points covered in this review are condensed into Fig. 5.

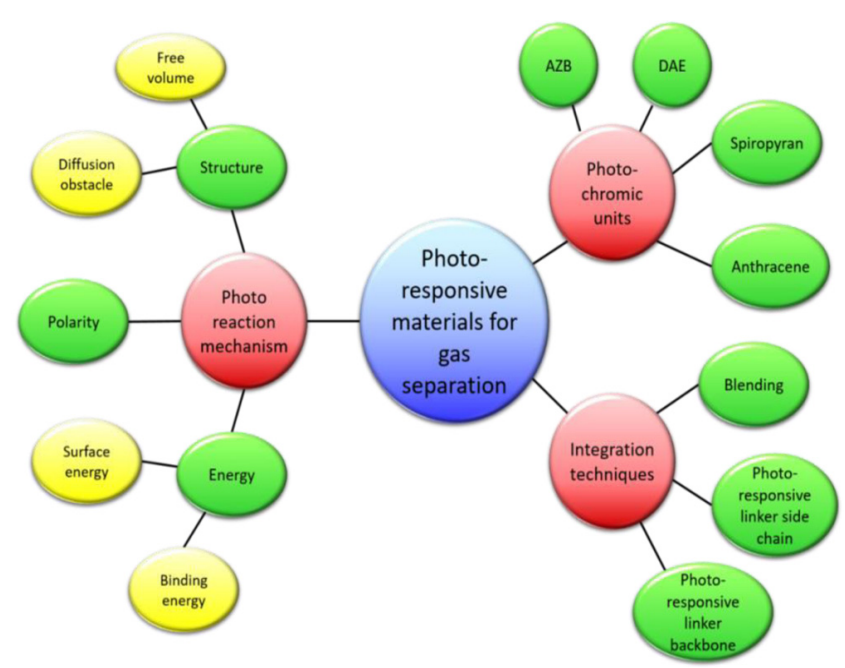

Fig. 5. Summary of photo-responsive materials for gas separation (Authors' own constructed figure).

\section{Conclusions and Future Direction}

This review has covered in detail the emerging use of photo-responsive materials for the specific purpose of gas separation. To help gain a better understanding of the research that has been done so far, the research to date has been explored from the aspects of photo-chromic units, their mechanism, their integration into the system, and the practicability for gas separation processes. While some work on gas separation through absorption or adsorption processes using photo-responsive materials are available, from this review it is evident that the investigation has mostly focused on two photo-chromic groups; AZB and DAE. In addition, most of the research found incorporated the photo-chromic units to MOF instead of membranes, and attached said groups to the dangling side chains instead of the backbone of the framework. It is clear that the research done so far have barely scratched the surface of the almost infinite possibilities those photo-responsive materials have to offer for gas separation processes.

Future direction of gas separation incorporating photo-responsive materials could be focused on overcoming the limitations of the process, such as the degradation of photo-chromic units, and potentially damaging effects of the UV lighting. Even though the results of photo-responsive materials has been promising so far to separate gases, the fact that so little research has been done on photo-chromic units other than AZB and DAE shows that the scope of this topic can be expanded. By pushing beyond the current 
boundaries placed on the photo-responsive materials for gas separation research, various improvements and innovations can be discovered.

\section{Acknowledgments}

This work is supported by MOHE Fundamental Research Grant Scheme FRGS/1/2017/TK02/USM/02/3 (203.PJKIMIA.6071367).

\section{References}

1. Shim J-G, Lee DW, Lee JH, Kwak N-S. Experimental study on capture of carbon dioxide and production of sodium bicarbonate from sodium hydroxide. Environ. Eng. Res. 2016;21:297-303.

2. Patkool C, Chawakitchareon P, Anuwattana R. Enhancement of efficiency of activated carbon impregnated chitosan for carbon dioxide adsorption. Environ. Eng. Res. 2014;19:289-292.

3. Rosli A, Ahmad AL, Lim JK, Low SC. Advances in liquid absorbents for $\mathrm{CO}_{2}$ capture: A Review. J. Phys. Sci. 2017;28:121.

4. Ahmad AL, Rosli A, Low SC, Lim JK. Effects of Silica loading on the absorption of carbon dioxide by mixed matrix membranes. J. Phys. Sci. 2018;29:91-97.

5. Dalane K, Dai Z, Mogseth G, Hillestad M, Deng L. Potential applications of membrane separation for subsea natural gas processing: A review. J. Nat. Gas Sci. Eng. 2017;39:101-117.

6. Guo Z, Zhang XM, Zhang CL, Luan JY. Research development of membrane materials for separation of $\mathrm{CO}_{2}$ from flue gas. Xian Dai Hua Gong. 2016;36:42-45\&47.

7. Hamid MRA, Jeong HK. Recent advances on mixed-matrix membranes for gas separation: Opportunities and engineering challenges. Korean J. Chem. Eng. 2018;35:1577-1600.

8. Sasikumar B, Arthanareeswaran G, Ismail AF. Recent progress in ionic liquid membranes for gas separation. J. Mol. Liq. 2018;266:330-341.

9. Tan PC, Ooi BS, Ahmad AL, Low SC. Monomer atomic configuration as key feature in governing the gas transport behaviors of polyimide membrane. J. Appl. Polym. Sci. 2018;135:46073.

10. Rosli A, Shoparwe NF, Ahmad AL, Low SC, Lim JK. Dynamic modelling and experimental validation of $\mathrm{CO}_{2}$ removal using hydrophobic membrane contactor with different types of absorbent. Sep. Purif. Technol. 2019;219:230-240.

11. Rosli A, Ahmad AL, Low SC. Anti-wetting polyvinylidene fluoride membrane incorporated with hydrophobic polyethylene-functionalized-silica to improve $\mathrm{CO}_{2}$ removal in membrane gas absorption. Sep. Purif. Technol. 2019;221:275-285.

12. Xiao Y, Low BT, Hosseini SS, Chung TS, Paul DR. The strategies of molecular architecture and modification of polyimide-based membranes for $\mathrm{CO}_{2}$ removal from natural gas - A review. Prog. Polym. Sci. 2009;34:561-580.

13. Li H, Chen V, Hou J, Dong G. Evaluation of $\mathrm{CO}_{2}$ capture with high performance hollow fibre membranes from flue gas: Final report. ANLEC report. Canberra: Cooperative research centre for greenhouse gas technologie; 2015.

14. Scholes CA, Tao WX, Stevens GW, Kentish SE. Sorption of methane, nitrogen, carbon dioxide, and water in Matrimid 5218.
J. Appl. Polym. Sci. 2010;117:2284-2289.

15. Wandera D, Wickramasinghe SR, Husson SM. Stimuli-responsive membranes. J. Membr. Sci. 2010;357:6-35.

16. Brunetti A, Macedonio F, Barbieri G, Drioli E. Membrane engineering for environmental protection and sustainable industrial growth: Options for water and gas treatment. Environ. Eng. Res. 2015;20:307-328.

17. Ohya H, Kudryavtsev VV, Semenova SI. Polyimide membranes: Applications, fabrications, and properties. Tokyo: Kodansha Ltd; 1996.

18. Kanj AB, Müller K, Heinke L. Stimuli-responsive metal-organic frameworks with photoswitchable azobenzene side groups. Macromol Rapid Commun. 2018;39:1700239.

19. Kausar A. Research progress in frontiers of poly (ionic liquid)s: A review. Polym. Plast. Technol. Eng. 2017;56:1823-1838.

20. He D, Susanto H, Ulbricht M. Photo-irradiation for preparation, modification and stimulation of polymeric membranes. Prog. Polym. Sci. 2009;34:62-98.

21. Becker D, Konnertz N, Böhning M, Schmidt J, Thomas A. Light-switchable polymers of intrinsic microporosity. Chem. Mater. 2016;28:8523-8529.

22. Schneemann A, Bon V, Schwedler I, et al. Flexible metal-organic frameworks. Chem. Soc. Rev. 2014;43:6062-6096.

23. Ng QH, Lim JK, Ahmad AL, Ooi BS, Low SC. Efficacy evaluation of the antifouling magnetite-PES composite membrane through QCM-D and magnetophoretic filtration performances. Sep. Purif. Technol. 2014;132:138-148.

24. Fan CB, Le Gong L, Huang L, et al. Significant enhancement of $\mathrm{C}_{2} \mathrm{H}_{2} / \mathrm{C}_{2} \mathrm{H}_{4}$ separation by a photochromic diarylethene unit: A temperature and light responsive separation switch. Angew. Chem. Int. Ed. 2017;56:7900-7906.

25. Huang R, Hill MR, Babarao R, Medhekar NV. $\mathrm{CO}_{2}$ Adsorption in azobenzene functionalized stimuli responsive metal-organic frameworks. J. Phys. Chem. C. 2016;120:16658-16667.

26. Ng QH, Lim JK, Ahmad AL, Ooi BS, Low SC. Magnetic nanoparticles augmented composite membranes in removal of organic foulant through magnetic actuation. J. Membr. Sci. 2015;493:134-146.

27. Chu L, Xie R, Ju X. Stimuli-responsive membranes: Smart tools for controllable mass-transfer and separation processes. Chin. J. Chem. Eng. 2011;19:891-903.

28. Ueki T. Stimuli-responsive polymers in ionic liquids. Polym. J. 2014;46:646-655

29. Bassanetti I, Bracco S, Comotti A, et al. Flexible porous molecular materials responsive to $\mathrm{CO}_{2}, \mathrm{CH}_{4}$ and Xe stimuli. J. Mater. Chem. A. 2018;6:14231-14239.

30. Gao Q, Xu J, Cao D, Chang Z, Bu X-H. A rigid nested metalOrganic framework featuring a thermoresponsive gating effect dominated by counterions. Angew. Chem. Int. Ed. 2016;55: 15027-15030.

31. Zhang Q, Zhang J, Wan S, Wang W, Fu L. Stimuli-responsive 2D materials beyond graphene. Adv. Funct. Mater. 2018;28: 1802500

32. Yanai N, Uemura T, Inoue M, et al. Guest-to-host transmission of structural changes for stimuli-responsive adsorption property. J. Am. Chem. Soc. 2012;134:4501-4504.

33. Ng QH, Lim JK, Ahmad AL, Low SC. Stability and fouling 
mechanism of magnetophoretic-actuated PES composite membrane in pH-dependent aqueous medium. J. Membr. Sci. 2016;508:40-50.

34. Nagarkar SS, Desai AV, Ghosh SK. Stimulus-responsive metalOrganic frameworks. Chem. Asian J. 2014;9:2358-2376.

35. Knebel A, Sundermann L, Mohmeyer A, et al. Azobenzene guest molecules as light-switchable $\mathrm{CO}_{2}$ valves in an ultrathin UiO-67 membrane. Chem. Mat. 2017;29:3111-3117.

36. Gui B, Meng Y, Xie Y, et al. Immobilizing organic-based molecular switches into metal-organic frameworks: A promising strategy for switching in solid state. Macromol. Rapid Commun. 2018;39:1700388.

37. Park J, Yuan D, Pham KT, et al. Reversible alteration of $\mathrm{CO}_{2}$ adsorption upon photochemical or thermal treatment in a metalOrganic framework. J. Am. Chem. Soc. 2012;134:99-102.

38. Weh K, Noack M, Hoffmann K, Schröder K-P, Caro J. Change of gas permeation by photoinduced switching of zeolite-azobenzene membranes of type MFI and FAU. Micropor. Mesopor. Mater. 2002;54:15-26.

39. Prasetya N, Ladewig BP. New Azo-DMOF-1 MOF as a photoresponsive low-energy $\mathrm{CO}_{2}$ adsorbent and its exceptional $\mathrm{CO}_{2} / \mathrm{N}_{2}$ separation performance in mixed matrix membranes. ACS Appl. Mater. Interfaces. 2018;10:34291-34301.

40. Ma S, Sun D, Wang X-S, Zhou H-C. A mesh-adjustable molecular sieve for general use in gas separation. Angew. Chem. Int. Ed. 2007;46:2458-2462.

41. Ma S, Sun D, Yuan D, Wang X-S, Zhou H-C. Preparation and gas adsorption studies of three mesh-adjustable molecular sieves with a common structure. J. Am. Chem. Soc. 2009;131: 6445-6451.

42. Wriedt M, Sculley JP, Yakovenko AA, et al. Low-energy selective capture of carbon dioxide by a pre-designed elastic single-molecule trap. Angew. Chem. Int. Ed. 2012;51:9804-9808.

43. Yang CT, Kshirsagar AR, Eddin AC, Lin LC, Poloni R. Tuning gas adsorption by metal node blocking in photoresponsive metal -Organic frameworks. Chem. Eur. J. 2018;24:15167-15172.

44. Gong LL, Feng XF, Luo F. Novel azo-metal-organic framework showing a 10-connected bct Net, breathing behavior, and unique photoswitching behavior toward $\mathrm{CO}_{2}$. Inorg. Chem. 2015;54: 11587-11589.

45. Lyndon R, Konstas K, Ladewig BP, et al. Dynamic photo-switching in metal-Organic frameworks as a route to low-energy carbon dioxide capture and release. Angew. Chem. 2013;125:3783-3786.

46. An W, Aulakh D, Zhang X, et al. Switching of adsorption properties in a zwitterionic metal-Organic framework triggered by photogenerated radical triplets. Chem. Mater. 2016;28:7825-7832.

47. Ding X, Han B-H. Metallophthalocyanine-based conjugated microporous polymers as highly efficient photosensitizers for singlet oxygen generation. Angew. Chem. 2015;127:6636-6639.

48. Castellanos S, Goulet-Hanssens A, Zhao F, et al. Structural effects in visible-light-responsive metal-organic frameworks incorporating ortho-fluoroazobenzenes. Chem. Eur. J. 2016;22: 746-752.

49. Tian H, Yang S. Recent progresses on diarylethene based photochromic switches. Chem. Soc. Rev. 2004;33:85-97.

50. Weh K, Noack M, Ruhmann R, et al. Modification of the transport properties of a polymethacrylate-azobenzene membrane by pho- tochemical switching. Chem. Eng. Technol. 1998;21:408-412. 51. Fan CB, Liu ZQ, Gong LL, et al. Photoswitching adsorption selectivity in a diarylethene-azobenzene MOF. Chem. Commun. 2017;53:763-766.

52. Kameda M, Sumaru K, Kanamori T, Shinbo T. Photoresponse gas permeability of azobenzene-functionalized glassy polymer films. J. Appl. Polym. Sci. 2003;88:2068-2072.

53. Li X, Li B, He M, et al. Convenient and robust route to photoswitchable hierarchical liquid crystal polymer stripes via flow-enabled self-assembly. ACS Appl. Mater. Interf. 2018;10: 4961-4970.

54. Zhu Y, Zhang W. Reversible tuning of pore size and $\mathrm{CO}_{2}$ adsorption in azobenzene functionalized porous organic polymers. Chem. Sci. 2014;5:4957-4961.

55. Nicoletta FP, Cupelli D, Formoso P, et al. Light responsive polymer membranes: A review. Membranes 2012;2:134-197.

56. Klajn R. Immobilized azobenzenes for the construction of photoresponsive materials. Pure Appl. Chem. 2010;82:2247-2279.

57. Modrow A, Zargarani D, Herges R, Stock N. Introducing a photo-switchable azo-functionality inside Cr-MIL-101-NH 2 by covalent post-synthetic modification. Dalton Trans. 2012;41: 8690-8696.

58. Luo F, Fan CB, Luo MB, et al. Photoswitching $\mathrm{CO}_{2}$ capture and release in a photochromic diarylethene metal-organic framework. Angew. Chem. Int. Ed. 2014;53:9298-9301.

59. Irie M. Diarylethenes for memories and switches. Chem. Rev. 2000;100:1685-1716.

60. Healey K, Liang W, Southon PD, Church TL, D'Alessandro DM. Photoresponsive spiropyran-functionalised MOF-808: Postsynthetic incorporation and light dependent gas adsorption properties. J. Mater. Chem. A. 2016;4:10816-10819.

61. Minkin VI. Photo-, thermo-, solvato-, and electrochromic spiroheterocyclic compounds. Chem. Rev. 2004;104:2751-2776.

62. Berkovic G, Krongauz V, Weiss V. Spiropyrans and spirooxazines for memories and switches. Chem. Rev. 2000;100: 1741-1754.

63. Huang N, Ding X, Kim J, Ihee H, Jiang D. A photoresponsive smart covalent organic framework. Angew. Chem. Int. Ed. 2015;54:8704-8707.

64. Li H, Sadiq MM, Suzuki K, et al. MaLISA - A cooperative method to release adsorbed gases from metal-organic frameworks. J. Mater. Chem. A. 2016;4:18757-18762.

65. Coudert Fo-X. Responsive metal-Organic frameworks and framework materials: Under pressure, taking the heat, in the spotlight, with friends. Chem. Mater. 2015;27:1905-1916.

66. Dang L-L, Zhang X-J, Zhang L, et al. Photo-responsive azo MOF exhibiting high selectivity for $\mathrm{CO}_{2}$ and xylene isomers. J. Coord. Chem. 2016;69:1179-1187.

67. Hermann D, Emerich H, Lepski R, Schaniel D, Ruschewitz U. Metal-organic frameworks as hosts for photochromic guest molecules. Inorg. Chem. 2013;52:2744-2749.

68. Li H, Martinez MR, Perry Z, et al. A robust metal-Organic framework for dynamic light-induced swing adsorption of carbon dioxide. Chem. Eur. J. 2016;22:11176-11179.

69. Zhang J, Wang L, Li N, et al. A novel azobenzene covalent organic framework. Cryst. Eng. Commun. 2014;16:6547-6551.

70. Tang H, Lu D, Wu C. Intramolecular hydrogen bonds enhance 
disparity in reactivity between isomers of photoswitchable sorbents and $\mathrm{CO}_{2}$ : A computational study. Chem. Phys. Chem. 2015;16:1926-1932.

71. Ulbricht M. Advanced functional polymer membranes. Polymer 2006;47:2217-2262.

72. Wang Z, Knebel A, Grosjean S, et al. Tunable molecular separation by nanoporous membranes. Nat. Commun. 2016;7:13872.

73. Fan H, Mundstock A, Feldhoff A, et al. Covalent organic framework-covalent organic framework bilayer membranes for highly selective gas separation. J. Am. Chem. Soc. 2018;140:10094-10098.

74. Duan K, Wang J, Zhang Y, Liu J. Covalent organic frameworks (COFs) functionalized mixed matrix membrane for effective
$\mathrm{CO}_{2} / \mathrm{N}_{2}$ separation. J. Membr. Sci. 2019;572:588-595.

75. Shan M, Seoane B, Andres-Garcia E, Kapteijn F, Gascon J. Mixed-matrix membranes containing an azine-linked covalent organic framework: Influence of the polymeric matrix on post-combustion $\mathrm{CO}_{2}$-capture. J. Membr. Sci. 2018;549:377-384.

76. Müller K, Knebel A, Zhao F, et al. Switching thin films of azobenzene-containing metal-organic frameworks with visible light. Chem. Eur. J. 2017;23:5434-5438.

77. Prasetya N, Teck AA, Ladewig BP. Matrimid-JUC-62 and Matrimid-PCN-250 mixed matrix membranes displaying light-responsive gas separation and beneficial ageing characteristics for $\mathrm{CO}_{2} / \mathrm{N}_{2}$ separation. Sci. Rep. 2018;8:2944. 